\title{
Development of dental resin luting agents based on Bis-EMA4: bond strength evaluation
}

\author{
R. R. Moraes $^{1 *}$, L. S. Gonçalves ${ }^{1}$, F. A. Ogliari $^{2}$, E. Piva $^{2}$, M. A. Sinhoreti ${ }^{1}$, L. Correr-Sobrinho $^{1}$ \\ ${ }^{1}$ Department of Restorative Dentistry, Dental Materials Division Piracicaba Dental School, \\ State University of Campinas Av. Limeira, 901 - 13414-903 - Piracicaba, SP, Brazil \\ 2Department of Restorative Dentistry School of Dentistry, Federal University of Pelotas R. Gonçalves Chaves, \\ 457 - 96015-560 - Pelotas, RS, Brazil
}

Received 25 September 2007; accepted in revised form 11 December 2007

\begin{abstract}
The aim of this study was to investigate the influence of incorporating Bis-EMA4 monomer into experimental Bis-GMA/TEGDMA-based resin luting agents on the bond strength to dentin. Seven mixtures were prepared with the following ratios (wt\%) of Bis-GMA/TEGDMA/Bis-EMA4: 50/50/0, 50/30/20, 50/10/40, 50/0/50, 30/10/60, 10/10/80 and 0/0/100. Camphorquinone (0.4 wt $\%)$, N,N-dimethyl-p-toluidine $(0.8 \mathrm{wt} \%)$ and hydroquinone $(0.2 \mathrm{wt} \%)$ were dissolved in each mixture, which was loaded with silanated strontium glass fillers to a constant content of $60 \mathrm{wt} \%$. Bond strength was evaluated by microshear testing $(n=10)$ on bovine dentin. Data were submitted to Analysis of Variance $(p<0.05)$. Modes of failure were classified under magnification (200x). Bond strength means (MPa), respective to each agent, were: 19.4, 19.8, 20.0, 19.1, 16.8, 18.7 and 17.8. No significant differences were detected among groups. Mixed failures were generally predominant for all materials. In conclusion, the addition of Bis-EMA4 presented no significant influence on the bond strength of the experimental resin luting agents to dentin.
\end{abstract}

Keywords: adhesion, dental materials, dentin, resin luting agents

\section{Introduction}

Over the past decade, the synthesis and characterization of inorganic-organic hybrid materials have received considerable attention [1]. In dentistry, the use of hybrid materials such as resin composites and luting agents is increasingly popular, mainly due to their aesthetics, low solubility and ability to bond to tooth structure. Formulations are generally a mixture of mono and dimethacrylate monomers loaded with silanated glass filler particles. Bisphenol A glycol dimethacrylate (Bis-GMA) is the most common monomer in the resin phase, presenting high molecular weight and low polymerization shrinkage [2]. Due to its high viscosity, the BisGMA is usually diluted with triethylene glycol dimethacrylate (TEGDMA) [2]. However,
TEGDMA has been linked to increased water sorption and polymerization shrinkage [2-4].

In order to overcome the drawbacks of the dilution process using TEGDMA, ethoxylated bisphenol A glycol dimethacrylate (Bis-EMA) has been investigated as an alternative monomer [5-6]. This is structurally analogous to Bis-GMA, but without the two pendant hydroxyl groups responsible for the high viscosity and water affinity of Bis-GMA. Therefore, the addition of Bis-EMA could minimize or eliminate the use of TEGDMA as a diluent comonomer, while potentially reducing the polymerization shrinkage and stress due to the higher molecular weight and lower mobility of Bis-EMA molecule compared with TEGDMA. 
Previous studies reported that the monomers composing the organic phase might affect the properties of a composite, such as its viscosity, degree of conversion and curing shrinkage [2-4, 6, 7]. In addition, as distinct monomers may present different wettability, shrinkage stress and double bond conversion values, their bond ability to tooth structures, mediated by an adhesive system, might be different. However, the effect of Bis-EMA addition on the bond strength of resin composites to dentin is not well-established.

Therefore, the aim of this study was to investigate the bond strength to dentin of experimental luting agents in which Bis-EMA4 replaced Bis-GMA and/or TEGDMA. The null hypothesis was that no significant difference in bond strength would be observed, irrespective of the monomers composing the luting materials.

\section{Experimental}

Seven experimental resin luting agents based on Bis-GMA, TEGDMA and/or Bis-EMA4 (Esstech Inc., Essington, PA, USA), were tested. The chemical structure of each monomer is shown in Figure 1. Bis-EMA4 is analogous to Bis-GMA; however, the former has four ethylene oxide units substituting the two hydroxyl groups between the aromatic backbone and the insaturates. The formulation of all luting agents is shown in Figure 2.

Starting from a 50:50 wt:wt ratio blend of BisGMA and TEGDMA, different mixtures were produced in which Bis-GMA and/or TEGDMA were substituted by Bis-EMA4.

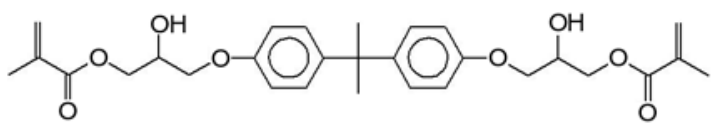

Bis-GMA
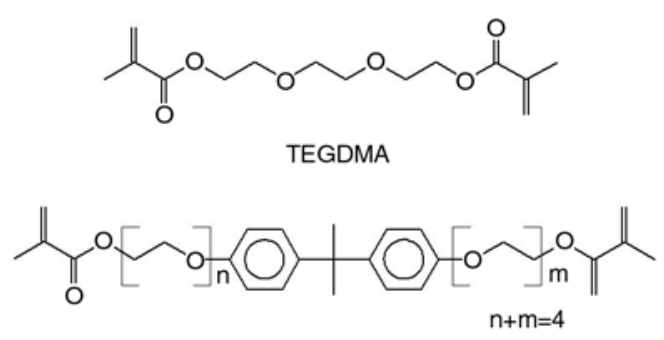

Bis-EMA4

Figure 1. Molecular structure of the monomers used in the study

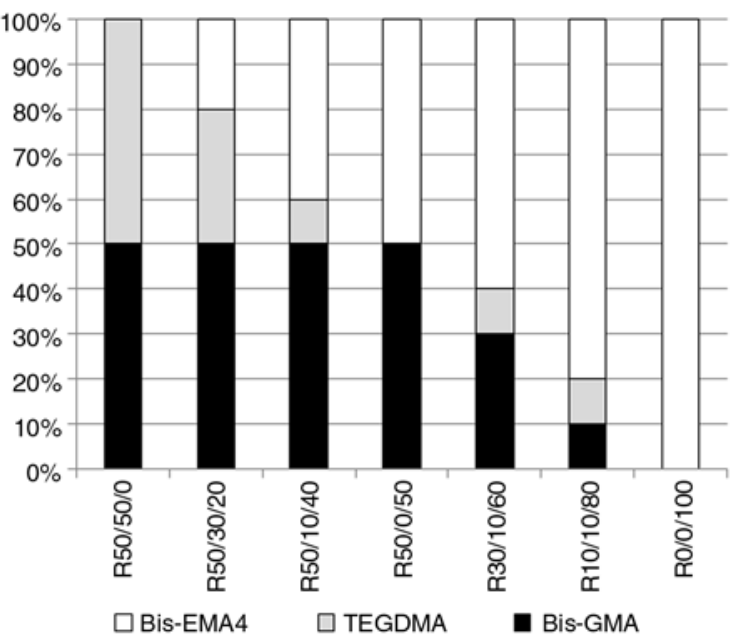

Figure 2. Formulation of the materials tested in the study

In order to make light-curing materials, $0.4 \mathrm{wt} \%$ of camphorquinone (Esstech Inc.) and $0.8 \mathrm{wt} \%$ of N,N-dimethyl-p-toluidine (Aldrich Chemical Co., Milwaukee, WI, USA) were dissolved in each mixture as photo-initiators. The proprietary monomers used in the study present an average of $0.03 \%$ of inhibitor. However, as a photo-sensitive curing initiation system was added to the mixtures, $0.2 \mathrm{wt} \%$ of hydroquinone (Aldrich) was used as a radical scavenger to increase the shelf-life of the materials. All chemicals were used as received, without further purification.

Materials were loaded with silanated strontium glass fillers (Esstech Inc.), 0.7 and $2 \mu \mathrm{m}$ in size, to a constant content of $60 \mathrm{wt} \%$ (1:1 of each size). Each material was labeled as $R(G / T / E)$, where $G=$ BisGMA wt $\%, \mathrm{~T}=$ TEGDMA wt $\%$ and $\mathrm{E}=$ BisEMA4 wt $\%$. The percentage values are relative to the monomer fraction in the organic phase.

For bond strength evaluation, bovine incisors were obtained, cleaned and stored in $0.5 \%$ chloramine-T solution for seven days. The teeth were then embedded in epoxy resin and their buccal faces were wet-ground with 180-, 220-, 400- and 600-grit $\mathrm{SiC}$ abrasive papers, in order to create a smooth, flat surface in medium dentin. The adhesive system Single Bond 2 (3M ESPE, St. Paul, MN, USA) was applied on dentin, according to the manufacturer's instructions. Absorbent paper was used to remove the excess dentin moisture.

In order to obtain specimens for the microshear bond strength test, the experimental set-up shown in Figure 3 was carried out [8]. Customized $0.5 \mathrm{~mm}$-thick elastomer molds, each with three 


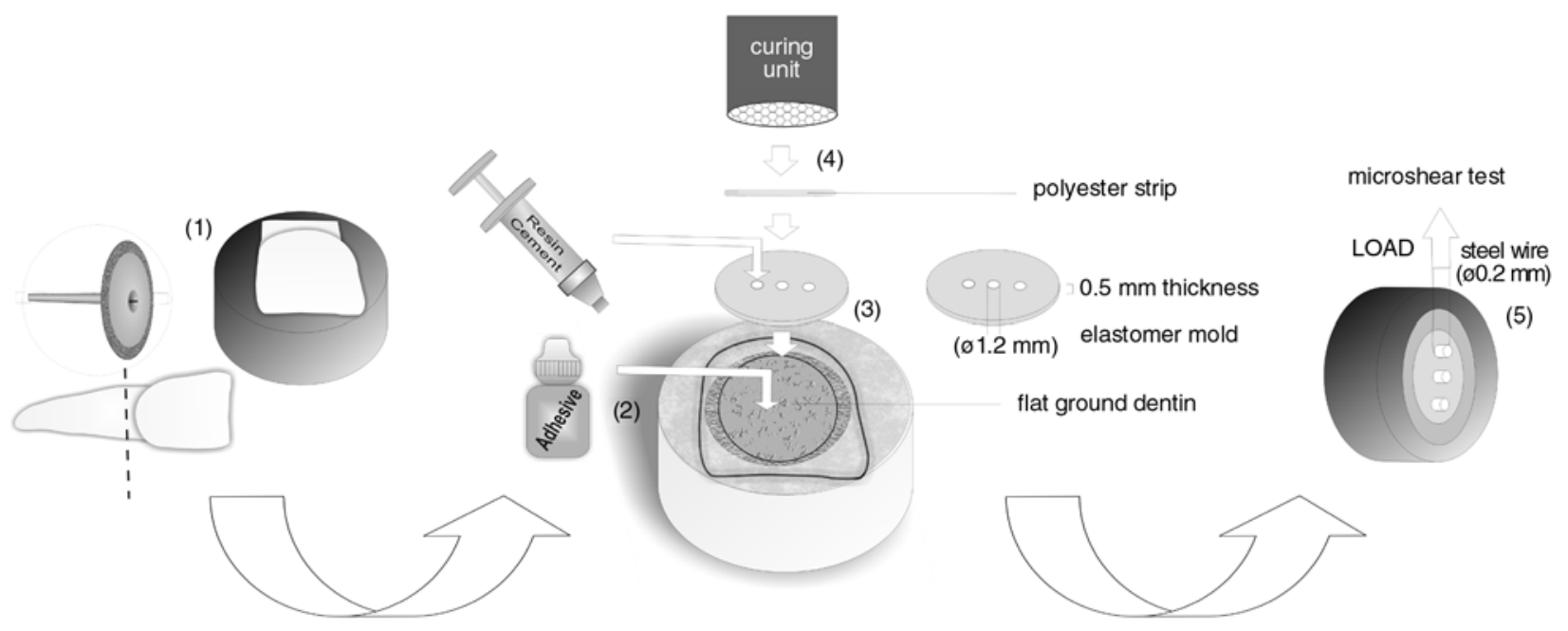

Figure 3. Experimental set-up for the bond strength evaluation: (1) bovine teeth embedded in epoxy resin; (2) bonding agent applied to ground dentin; (3) elastomer mold with cylinder-shaped orifices positioned onto the surface and filled with luting agent; (4) polyester strip placed between the mold and the light guide tip; (5) microshear bond strength test carried out

cylinder-shaped orifices (1.2 $\mathrm{mm}$ in diameter), were placed in the teeth surfaces, allowing delimitation of the bonding area. After photo-activation of the bonding agent (quartz-tungsten-halogen lightcuring unit XL2500; 3M ESPE), the orifices were filled with each experimental luting agent, and a transparent polyester strip was placed over the filled orifices. Prior to the curing procedures, a constant and uniform $250 \mathrm{~g}$ cementation load was applied for $2 \mathrm{~min}$, using a custom-made device. The output irradiance of the curing unit was $700 \mathrm{~mW} / \mathrm{cm}^{2}$, between 410 and $510 \mathrm{~nm}$, confirmed with a digital power meter (Ophir Optronics, Danvers, MA, USA) and a computer-controlled spectrometer (USB 2000; Ocean Optics, Dunedin, FL, USA), respectively.

After storing the samples in distilled water at $37^{\circ} \mathrm{C}$ for $24 \mathrm{~h}$, all resin cylinders were checked under magnification (40x): those presenting flaws, irregularities or bonding defects were eliminated. For the microshear test, a thin steel wire $(0.2 \mathrm{~mm}$ in diameter) was looped around each cylinder and aligned with the bonding interface. The test was conducted in a universal testing machine (model 4411; Instron Inc., Canton, MA, USA), at a crosshead speed of $0.5 \mathrm{~mm} / \mathrm{min}$ until failure. Bond strength values were calculated in MPa. For each group, 10 specimens were tested, and the average value of the three resin cylinders was recorded as the bond strength for each sample. Bond strength data were submitted to one-way Analysis of Variance $(p=0.05)$.

The fractured specimens were examined under optical microscopy at a $200 \times$ magnification. Modes of failure were classified as follows: adhesive failure (Mode 1), cohesive failure within dentin (Mode 2), or mixed failure involving bonding agent, luting material and dentin (Mode 3). Additionally, representative fractured specimens were coated with gold and examined under scanning electron microscopy (SEM - JSM5600LV; Jeol Inc., Peabody, MA, USA).

\section{Results}

Results for the microshear test are shown in Table 1. The Analysis of Variance showed that,

Table 1. Means (standard deviations) for bond strength to dentin, and percentage of scores for the failure analysis

\begin{tabular}{|l|c|c|c|c|}
\hline \multirow{2}{*}{ Material } & Bond & \multicolumn{3}{|c|}{ Failure modes*[\%] } \\
\cline { 3 - 5 } & $\begin{array}{c}\text { strength } \\
{[\mathbf{M P a}]}\end{array}$ & Mode 1 & Mode 2 & Mode 3 \\
\cline { 3 - 5 } & $19.4(4.3)$ & 14.3 & 33.3 & 52.4 \\
\hline $\mathrm{R} 50 / 50 / 0$ & $19.8(5.6)$ & 27.8 & 18.7 & 53.5 \\
\hline $\mathrm{R} 50 / 30 / 20$ & $20.0(6.7)$ & 12.5 & 12.5 & 75.0 \\
\hline $\mathrm{R} 50 / 10 / 40$ & $19.1(4.3)$ & 64.8 & 0 & 35.2 \\
\hline $\mathrm{R} 50 / 0 / 50$ & $16.8(4.2)$ & 25.9 & 18.5 & 55.6 \\
\hline $\mathrm{R} 30 / 10 / 60 / 10 / 80$ & $18.7(5.3)$ & 30.4 & 13.0 & 56.6 \\
\hline $\mathrm{R} 0 / 0 / 100$ & $17.8(3.6)$ & 32.0 & 12.0 & 56.0 \\
\hline
\end{tabular}

*Mode 1: adhesive failure; Mode 2: cohesive failure within dentin, Mode 3: mixed failure involving bonding agent, luting material and dentin 

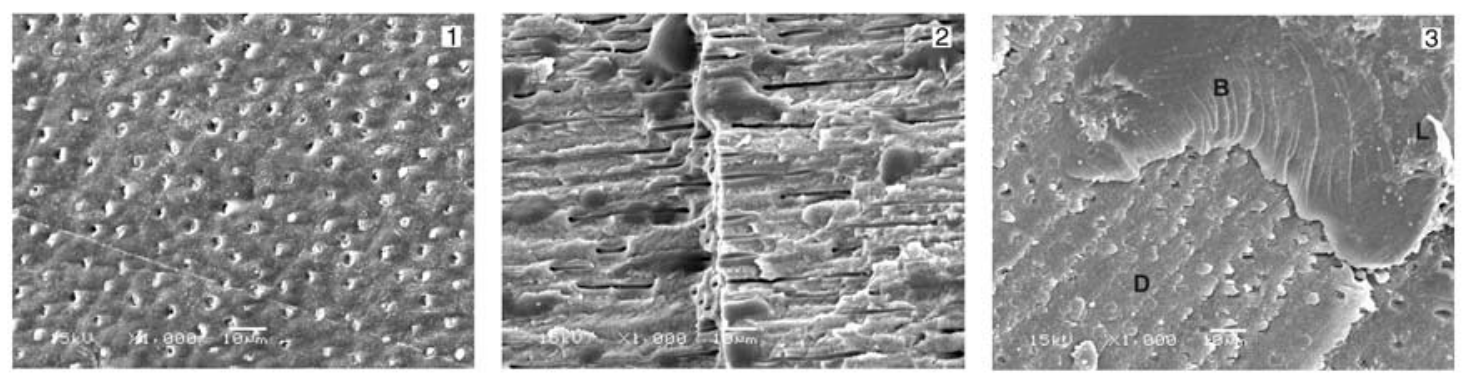

Figure 4. Representative SEM images of fractured specimens. (1) adhesive failure, which is characterized by the presence of opened and partially opened dentinal tubules and scratch lines formed during grounding procedures; (2) cohesive failure within dentin; (3) mixed failure involving bonding agent (B), luting material (L) and dentin (D)

irrespective of the monomers composing the organic phase of the luting agents, no significant differences in bond strength were detected $(p=$ 0.778). Table 1 also shows the results for the failure analysis. SEM pictures of fractured specimens are shown in Figure 4. A predominance of mixed failures (Mode 3) was generally detected for all materials, except for R50/0/50, for which a predominance of adhesive failures (Mode 1) was detected. Cohesive failures within the luting agent were not observed, irrespective of the material tested.

\section{Discussion}

The current outcomes showed similar microshear bond strengths to dentin for the different resin luting agents. Therefore, the null hypothesis was accepted. The distinct monomers present different molecular weight, chain flexibility and curing reactivity [2, 5-7], implying that several properties of a composite might be affected by its components [9]. Although the bonding ability to tooth substrate could also be influenced by the composition of the agents [10], the current outcomes showed no significant effect.

For a resin-based composite, the main properties that might affect its bonding ability to tooth tissues are the degree of conversion, wetability and shrinkage stress. In fact, $\mathrm{Xu}$ et al. [11] reported that the higher the double bond conversion of a composite, the higher its bond strength to dentin. This might result from the better mechanical properties disclosed by more properly cured materials [9], which could enhance the bonding to dentin. Previous tests with the same experimental luting agents tested here showed differences in the degree of conversion among materials [12]. Furthermore, during the mixing procedures for preparing the luting agents, replacing TEGDMA with Bis-EMA4 increased the apparent viscosity of the agents. Although both of these monomers are less viscous than Bis-GMA, this observation is related to the fact that BisEMA4 presents a higher molecular weight and structure stiffness than TEGDMA. Materials with high viscosity might present poor wettability with the substrate surface, potentially interfering with the bonding outcomes.

Moreover, differences in the resin component might interfere with the shrinkage stress and the interaction of force vectors created during constrained polymerization, which may produce effects detrimental to the material being bonded, as a competition between contraction and bond is created [13]. Nonetheless, despite the above mentioned characteristics, no significant differences in microshear bond strength were detected among the experimental materials, suggesting that the bonding ability to dentin is less influenced by the components of the resin phase than other properties of the polymer.

Although it is known that polymerization shrinkage is related to double bond conversion, it has been demonstrated that the shrinkage stress development of a resin material depends not only upon its mass and properties, but also on the configuration-factor (C-factor) $[14,15]$. The $\mathrm{C}$-factor refers to a ratio of bonded to unbonded surface area in a cavity preparation. For example, a box-like cavity has five bonded surfaces and one unbonded surface, giving it a C-factor of 5, assuming all walls have the same surface area. During the polymerization stage, the maturing composite-dentin bond strength competes with the shrinkage stress of the setting material [14]. In situations where shrinkage stress exceeds bonding strength, debonding of restorations might 
occur, posing a risk to post-operative sensitivity and secondary caries.

However, it is important to highlight that the experimental design of traditional bond strength tests allow the composite to be cured from a free surface, with low $\mathrm{C}$-factor, and having the polymerization shrinkage restricted to one direction could minimize the development of setting stresses [14]. Therefore, further investigations on the current materials are required. In clinical practice, this situation could be relevant during the cementation of an intra-radicular post, for instance, as a high Cfactor condition would be established.

Results for the failure analysis were in general similar for all materials. However, more adhesive failures were verified for R50/0/50, with no cohesive failures within dentin. This result is probably explained by the high viscosity of this luting material, which might have interfered with its wettability to the bonding system surface, resulting in increased adhesive failures. In summary, the current outcomes indicate that the monomers composing the resin phase of resin luting agents seem to present no significant influence on their bonding ability to bovine dentin. Nonetheless, other properties need to be evaluated in order to known the characteristics of polymers derived from different mixtures of monomers.

\section{Conclusions}

The addition of Bis-EMA4 into experimental luting agents based on Bis-GMA/TEGDMA presented no significant influence on the bond strength to dentin. From this standpoint, Bis-EMA4 demonstrated to be a useful alternative in the development of resin luting agents.

\section{Acknowledgements}

The first author is grateful to $\mathrm{CNPq} / \mathrm{Brazil}$ for a scholarship. Authors thank Esstech Inc. for the donation of the reagents.

\section{References}

[1] Da Z. L., Zhang Q. Q., Wu D. M., Yang D. Y., Qiu F. $\mathrm{X}$.: Synthesis, characterization and thermal properties of inorganic-organic hybrid. Express Polymer Letters, 1, 698-703 (2007).
[2] Anseth K. S., Goodner M. D., Reill M. A., Kannurpatti A. R., Newman S. M., Bowman C. N.: The influence of comonomer composition on dimethacrylate resin properties for dental composites. Journal of Dental Research, 75, 1607-1612 (1996).

[3] Dermann K., Rupp N. W., Brauer G. M.: Effect of hydrophilic diluents on the properties of cured composites. Journal of Dental Research, 61, 1250-1254 (1982).

[4] Dulik D., Bernier R., Brauer G. M.: Effect of diluent monomer on the physical-properties of Bis-GMAbased composites. Journal of Dental Research, 60, 983-989 (1981).

[5] Sideridou I., Achilias D. S., Spyroudi C., Karabela M.: Water sorption characteristics of light-cured dental resins and composites based on Bis-EMA/PCDMA. Biomaterials, 25, 367-376 (2004).

[6] Sideridou I., Tserki V., Papanastasiou G.: Effect of chemical structure on degree of conversion in lightcured dimethacrylate-based dental resins. Biomaterials, 23, 1819-1829 (2002).

[7] Stansbury J. W., Dickens S. H.: Network formation and compositional drift during photo-initiated copolymerization of dimethacrylate monomers. Polymer, 42, 6363-6369 (2001).

[8] Moraes R. R., Correr-Sobrinho L., Sinhoreti M. A., Puppin-Rontani R. M., Ogliari F. A., Piva E.: Lightactivation of resin cement through ceramic: relationship between irradiance intensity and bond strength to dentin. Journal of Biomedical Materials Research, Part B: Applied Biomaterials, in press, doi: 10.1002/ jbm.b.30928 (2007).

[9] Ferracane J. L., Greener E. H.: The effect of resin formulation on the degree of conversion and mechanical properties of dental restorative resins. Journal of Biomedical Materials Research, 20, 121-131 (1986).

[10] Thomsen K. B., Peutzfeldt A.: Resin composites: Strength of the bond to dentin versus mechanical properties. Clinical Oral Investigations, 11, 45-49 (2007).

[11] Xu X., Sandras D. A., Burgess J. O.: Shear bond strength with increasing light-guide distance from dentin. Journal of Esthetic and Restorative Dentistry, 18, 19-27 (2006).

[12] Moraes R. R., Ogliari F. A., Piva E., Petzhold C. L., Sinhoreti M. A. C., Correr-Sobrinho L.: Influence of Bis-EMA4 on properties of experimental resin luting agents. Brazilian Oral Research, 21, 194 (2007).

[13] Cabrera E., de la Macorra J. C.: Polymerization shrinkage influences microtensile bond strength. Journal of Dental Research, 86, 227-231 (2007).

[14] Feilzer A. J., De Gee A. J., Davidson C. L.: Setting stress in composite resin in relation to configuration of the restoration. Journal of Dental Research, 66, 16361639 (1987).

[15] Watts D. C., Satterthwaite J. D.: Axial shrinkagestress depends upon both $\mathrm{C}$-factor and composite mass. Dental Materials, 24, 1-8 (2008). 